\title{
Observations of a Correlated Gamma-Ray and Optical Flare for BL Lacertae
}

\author{
S. D. Bloom ${ }^{1}$, D. L. Bertsch, R. C. Hartman, P. Sreekumar ${ }^{2}$, and D. J. Thompson \\ Laboratory for High Energy Astrophysics, NASA/Goddard Space Flight Center, \\ Greenbelt, MD 20771 \\ T. J. Balonek, E. Beckerman ${ }^{3}$, S. M. Davis, K. Whitman ${ }^{4}$ \\ Department of Physics and Astronomy, Colgate University, Hamilton, NY 13346-1398 \\ H. R. Miller, D. Nair, L. C. Roberts, Jr. \\ Department of Physics and Astronomy, Georgia State University, Atlanta, GA 30303 \\ G. Tosti \\ Cattedra di Astrofisica, Universitá di Perugia, Via A. Pascoli, I-06100 Perugia, Italy \\ E. Massaro, R. Nesci, M. Maesano, F. Montagni \\ Istituto Astronomico, Universitá "La Sapienza", via G. M. Lancisi, 29, I-00161, Rome, Italy \\ M. Jang \\ Department of Astronomy and Space Science, Kyung Hee University, Yongin, Kyungki-Do, \\ 449-701, Korea
}

H. A. Bock, M. Dietrich, M. Herter, K. Otterbein, M. Pfeiffer, T. Seitz, S. Wagner Landessternwarte Heidelberg, Königstuhl, 69117 Heidelberg, Germany

Received ; accepted

\footnotetext{
${ }^{1}$ NAS/NRC Resident Research Associate

${ }^{2}$ Universities Space Research Association

${ }^{3}$ Visiting student from Wesleyan University

${ }^{4}$ Visiting student from Cornell University
} 


\begin{abstract}
BL Lacertae was detected by the EGRET instrument on CGRO at the 10.2 $\sigma$ level with an average flux of $171 \pm 42 \times 10^{-8}$ photons $\mathrm{cm}^{-2} \mathrm{~s}^{-1}$ at energies $>100 \mathrm{MeV}$ during the optical outburst of 1997 July. This flux is more than 4 times the previously highest level. Within the July 15-22 observation there was a dramatic factor of 2.5 increase in the gamma-ray flux on July 18.75-19.08, apparently preceding by several hours a brief optical flare. The gamma-ray flux decreased to its previous level within 8 hrs and the optical flux decreased to its prior level in less than 2 hrs. The gamma-ray photon spectral index of 1.68 \pm 0.12 indicates that the spectrum during the 7-day observation was harder than the previous detection.
\end{abstract}

Subject headings: Gamma Rays: observations — galaxies: active — BL Lacertae Objects: individual (BL Lacertae) 


\section{Introduction}

BL Lacertae $(z=0.069)$ is one of about 60 blazars that have been detected by the EGRET instrument on the Compton Gamma-Ray Observatory (CGRO) over the last 6 years (Hartman et al 1997). Though bright and variable across most of the electromagnetic spectrum, it has not been an outstanding gamma-ray source before this most recent outburst (Catanese et al 1997 and references therein). This object was first detected at gamma-ray energies greater than $100 \mathrm{MeV}$ by EGRET in 1995 (Catanese et al 1997 and see Figure 1) but prior to this, BL Lac gamma-ray observations only yielded upper limits. During a recent optical outburst of BL Lac (Noble et al 1997; Maesano et al 1997) the CGRO was re-pointed to observe the BL Lac flare in gamma-rays. BL Lac is the prototypcial object for a class of active galactic nuclei (AGN) which typically have very weak emission and absorption lines and are characterized by variabilty in continuum emission and polarization(Peterson 1997). Approximately $20 \%$ of those blazars detected by CGRO are BL Lac objects and the rest

are flat spectrum radio quasars (FSRQ's). Although some gamma-ray properties are similar for these two classes, BL Lac objects tend to have lower flux and less prominent variability than FSRQs (Mukherjee et al 1997).

The nature of the high energy gamma-ray emission of blazars has been disputed, with several models fitting data well for particular sources at specific times (eg. Hartman et al 1996). However, most processes discussed in the literature are of the inverse Compton type. Inverse Compton emission is a natural consequence of high soft photon densities in the vicinity of the relativistic electron populations of blazar jets. If the seed photons are created via synchrotron emission in the jet and then scatter off of the same population of electrons which produced them (synchrotron self Compton or SSC; see Bloom \&Marscher 1996), the gamma-rays will be observed to vary simultaneously with the low energy photons (submillimeter through optical). In the external scattering "mirror" model of Ghisellini 
\& Madau 1996 the optical outburst would lead the gamma-rays by roughly 1 day, since the optical emission is created via synchrotron emission within the jet and the optical photons are then scattered off of the broad line region clouds and back into the jet, where they are scattered finally by the relativistic electrons to produce high energy gamma-rays. The multiple relativistic Doppler boosts involved greatly enhance the observed gamma-ray emission. External scattering models which involve soft optical photons from an accretion disk (Dermer \& Schlickeiser 1993) are much harder to test, since it is likely that the optical spectra of blazars are dominated by synchrotron emission from the jet, which "washes out" the optical emission from an accretion disk. However, in this case one would also expect optical emission leading the gamma-ray emission.

Section 2 will summarize the gamma-ray and optical observations, and Section 3 will discuss the results in light of the processes mentioned above.

\section{Observations}

During 1997 July 15-22, the EGRET instrument observed the region centered on BL Lac in narrow field of view mode, which effectively looks at gamma-ray sources within 20 degrees of the CGRO pointing axis. Details of the EGRET instrument can be found in (Hartman et al 1992). Maps of the photon counts were created using the standard techniques. We initially broke up the 7-day total exposure into 7 -day maps, and then broken up the 4th 1-day map into 3 equal $8 \mathrm{hr}$ intervals once it was established that the gamma-ray emission peaked on the fourth day. A maximum likelihood analysis Mattox $e$ al 1996) was performed on the map to determine fluxes for individual sources, taking into account the galactic diffuse background model of Hunter et al 1997 and the extragalactic diffuse model of Sreekumar et al 1997. The gap of several hours seen between the points for day 3 and day 4 of the gamma-ray observations (Figure 2) was caused by a scheduled loss 
of telemetry.

Degradation of the EGRET spark chamber gas has led to a decreased efficiency in detecting gamma-rays, mainly due to the reduced capability of characterizing "good" gamma-ray events. Thus, scale factors have been applied to the current data so that they are calibrated consistently with data acquired at earlier points in the EGRET mission (see Esposito et al 1997 for details of the in-flight calibration). These scale factors are determined by taking the ratio of the average intensity of the diffuse emission from a well studied region of the sky (and at a given energy interval) from early in the mission and the diffuse emission from the same region at the later time of interest. Since the galactic diffuse emission is not expected to show any time variability, the diminished intensity is completely due to decreased instrument performance. Thus, these scale factors represent an accurate relative calibration of the EGRET data with time. Prior to this observation, a scale factor of 1.6 was calculated. We have now determined an additional scale factor of 2.02 (total scaling is $1.6 \times 2.02=3.2)$ with an uncertainty of $20 \%$ determined by recent analysis. Of course, the amplitude of this flare relative to previous measurements depends on the size of this correction, but this does not affect relative variations within a viewing period.

Visual magnitudes were determined by several amateur observers, and these results were retrieved from the American Association of Variable Star Observers (AAVSO) database (Mattei 1997) . Though all of the observers have used the same comparison stars, many magnitude determinations were made by eye and thus there may be some scatter in the measurements that is not intrinsic to the source (these measurements also have large error bars). However, in Figure 2, we see that there is agreement between visual magnitudes determined by eye, and those determined by a CCD. R magnitudes measured using the 16 inch telescope of Foggy Bottom Observatory (Colgate), the $0.4 \mathrm{~m}$ telescope at Georgia State University, the $0.4 \mathrm{~m}$ telescope at Perugia University Observatory, the $0.5 \mathrm{~m}$ 
telescope at Vallinfreda, Italy, the 0.7 meter telescope at Landessternwarte Heidelberg and the Kyung Hee University telescope are used along with $\mathrm{R}$ magnitudes from Ma \&Barry 1997. The $V-R$ color is approximately 0.7 , and we have applied a $\Delta \mathrm{m}$ correction of 0.7 to the AAVSO data and our $\mathrm{V}$ band data (from the Italian sites) to place them on the same scale as the $\mathrm{R}$ band data. We note that BL Lac was also detected by the OSSE instrument in the 50-300 keV range (Grove \&Johnson 1997) and was observed to vary in the X-ray range at 2-10 keV with both RXTE and ASCA(Makino et al 1997; Madejski et al 1997).

\section{Discussion}

From Figure 1 we can see clearly that the average gamma-ray flux is at least four times the previous value in 1995. Also, this measurement is twelve times greater than the summed $95 \%$ confidence upper limit for phases 1-3 (Catanese et al 1997). Though the precise peak of the gamma-ray flare is uncertain, Figure 2 suggests that there is a lag of several hours between the peak optical and peak gamma-ray measurements. Unfortunately, the sampling of the optical data does not completely rule out the possibility of a very rapid optical flare, of larger amplitude than the observed optical peak, occuring during the peak of the gamma-ray flare (or preceding it). However, the gamma-ray flux had already decreased by the time of the peak optical magnitude. We do note that since there are many other comparable optical flaring events during this time period, we can not be certain that the

peak of the gamma-ray outburst is physically related to this optical maximum. In addition, the exact value of the gamma-ray fluxes are somehwat dependent on the arbitrary binning used here. Comparable effects have been seen for other blazars. For the blazar 1406-076, the gamma-ray peak lagged the optical peak by one day (Wagner et al 1995). The 1991 June flare of 3C279 showed a similar effect (Hartman et al 1996). However, none of these observations had optical data as densely sampled as this most recent observation of BL Lac. 
In order to determine the best model for the high energy emission, the relative lags must be determined accurately.

One possibility is that the gamma-rays are created via SSC emission from electrons accelerated in a front, possibly a shock, moving through a jet Romanova \& Lovelace 1997: Marscher 1996) . However, if the optical emission lags the gamma-ray emission, then the optical flare can not be the source of soft photons for the gamma-ray flare unless the emitting region is initially optically thick to optical radiation. Such extreme opacity conditions have not been previously observed for outbursts of blazars. The near simultaneity of the gamma-ray and optical peaks (with the possibility of a delay of several hours) rules out external scattering models mentioned above. We also note that the ratio of the optical fluxes from this time period and that of January 1995 is about 4.8, and that the ratio of the gamma-ray fluxes is 4.2. A simple interpretation of the SSC process shows that equal enhancements of the magnetic field and relativistic electron density would lead to a greater amplitude of variation for the higher energy SSC flux, than for the synchrotron flux, due to the extra factor of $N_{O}$ (electron distribution normalization factor) in computing the SSC flux (Bloom \&Marscher 1996). However, if the Compton optical depth stays constant during the variations, the amplitudes of variation for both synchrotron and SSC would be identical.

Figure 3 shows that the gamma-ray spectrum was harder during this flare, as compared to that during the previous detection (a photon spectral index of $1.68 \pm 0.16$ for the recent detection and $2.27 \pm 0.30$ for the earlier observation (Catanese et al 1997)). Spectral hardening during gamma-ray flares has been seen previously for other blazars Sreekumar et al 1997; Mukherjee et al 1997) and is likely caused by injection of higher energy electrons which scatter soft photons preferentially to the higher energies of the EGRET range. It is possible that second order energy dependencies of the scale factor mentioned above could 
contribute, in part, to this spectral hardening.

To our knowledge, this is the first Target of Opportunity study of a blazar which has made extensive use of observations conducted by the international amatuer astronomy community. We suggest that other investigators make use of this valuable resource in future studies. We also wish to stress the importance of including optical monitoring telescopes on future X-ray and gamma-ray satellites, since it is likely that some of the confusion over time correlation of flares would be resolved by such instruments.

We thank J. Mattox for many useful comments and for setting up a World Wide Web home page supplying astronomers with up to date information on the BL Lac outburst. HRM and ADN are supported in part by GSU's Research Promotion and Enhancement Fund, and by grants from the Research Corporation, and NASA (NAGW-4397). 


\section{REFERENCES}

Bloom, S. D. \& Marscher, A. P. 1996, ApJ, 461, 657

Catanese, M. et al 1997, ApJ, 480, 562

Dermer, C. \& Schlickeiser, R., 1993, ApJ, 416, 458

Esposito, J. et al 1997, ApJS, submitted

Ghisellini, G. \& Madau 1996, MNRAS, 280, 67

Grove, J. E. \& Johnson, W. N. 1997, IAU Circular 6705

Hartman, R. C. et al 1992, in Proceedings of The Compton Observatory Science Worskshop, eds. C. R. Shrader, N. Gehrels,\&B. Dennis(NASA Conf. Publ. 3137), 116

Hartman, R. C. et al 1996, ApJ, 461, 698

Hartman, R. C. et al 1997, Proceedings of the 4th Compton Symposium, in press

Hunter, S. D. et al 1997, ApJ,481,205

Ma, Y. \& Barry, D. 1997, IAU Circular 6703

Madejski, G. et al 1997, IAU Circular 6705

Maesano, M. et al 1997, IAU Circular 6700

Makino et al 1997, IAU Circular 6708

Marscher, A. P. 1996, in Proceedings of the Heidleberg Workshop on Gamma-Ray Emitting AGN, eds. J. G. Kirk, M. Camenzind, C. von Montigny, S. Wagner (Heidelberg:MPIFK), p.103

Mattei, J. A., Observations from the AAVSO International Database, private communication 
Mattox, J. R. et al 1996, ApJ, 461, 396

Mukherjee, R. et al 1996, ApJ, 470,831

Mukherjee, R. et al 1997, ApJ, in press

Peterson, B. 1997, An Introduction to Active Galactic Nuclei (Cambridge:Cambridge University Press)

Noble, J. C. et al 1997, IAU Circular 6693

Romanova, M. M. \& Lovelace, R. V. E 1997, ApJ, 475, 97

Sreekumar, P. et al 1997, ApJ, submitted

Sreekumar, P. et al 1997, Proceedings of The 4th Compton Symposium, in press

Wagner, S. et al 1995, ApJ, 454, L97 
Fig. 1. - The complete time history of EGRET Gamma-ray Observations for BL Lacertae. Until Jan 1995, this source was not detected. The arrows represent $95 \%$ confidence (2 sigma) upper-limits

Fig. 2.- Optical and Gamma-Ray Light Curves for July 1997 Flare. Both plots show that there was a peak on July 19. On the upper plot, the open triangles represent visual magnitudes (recorded by eye) corrected for $V-R=0.7$, asterisks are $\mathrm{V}$ magntidues recorded by $\mathrm{CCD}$ with $\mathrm{V}$ filter, the open diamonds represent the $\mathrm{R}$ magnitudes of Ma \& Barry, and the filled circles are the $\mathrm{R}$ and $\mathrm{V}$ band data acquired by the authors at the sites mentioned in the text. The dashed vertical line crosssing both plots shows the end of the gamma-ray flare, for comparison to the optical flare.

Fig. 3.- Gamma-Ray Spectra for BL Lac. The most recent data (July 1997) suggests that the spectrum has hardened as compared to the earlier measurement of (January 1995) 


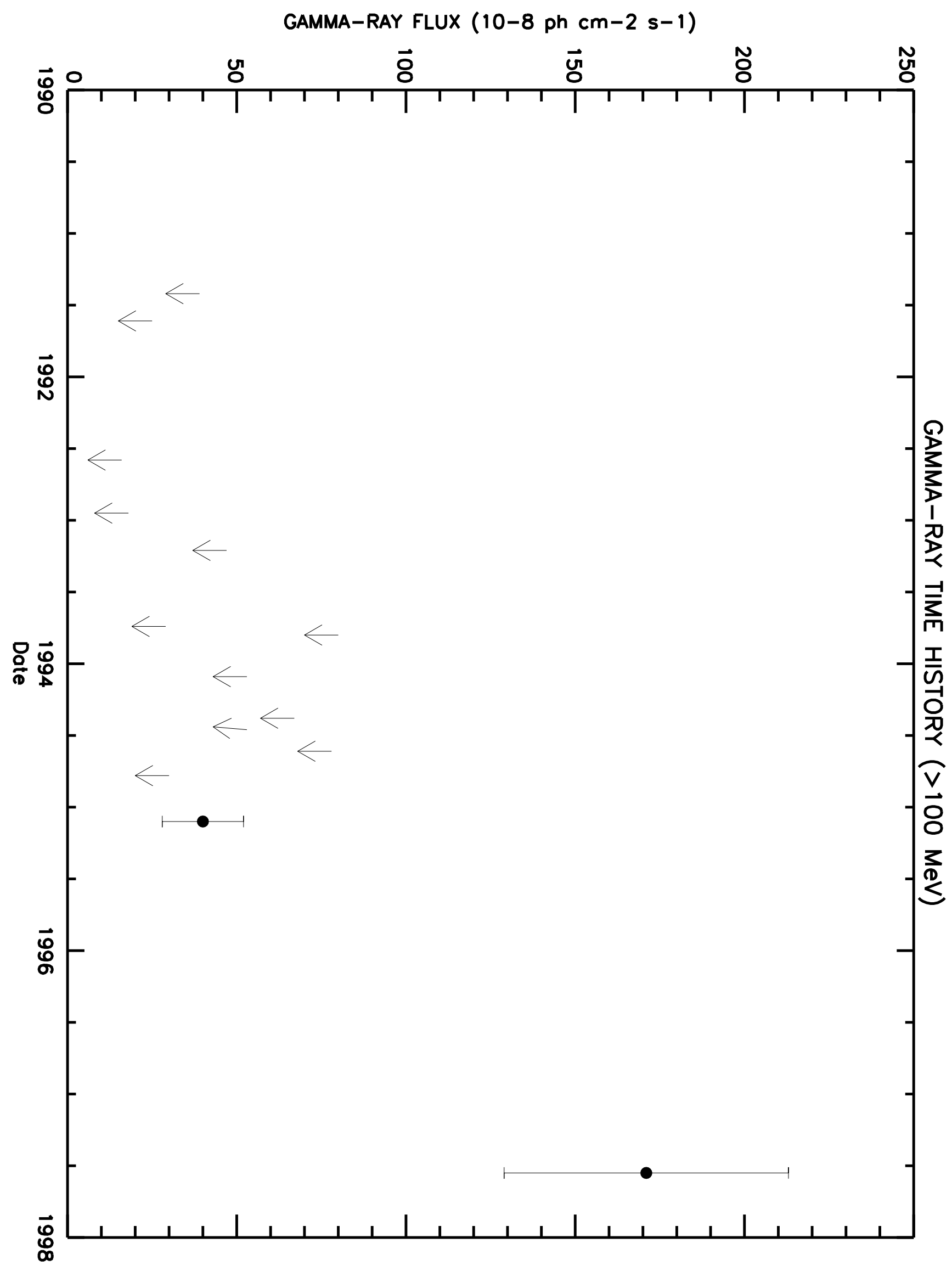









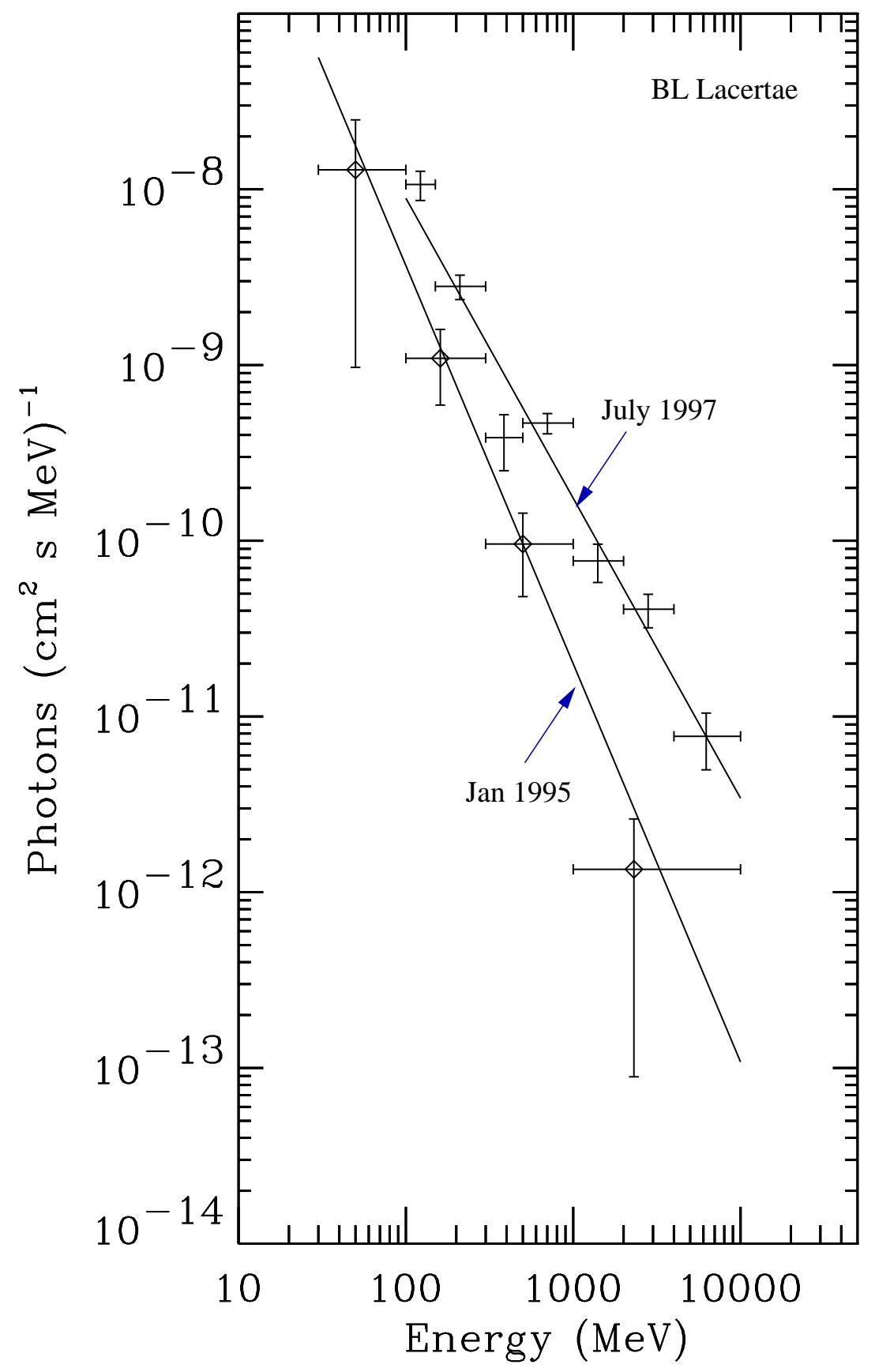

